\section{Eosinophil cytolysis and release of cell-free granules}

\author{
Helene F. Rosenberg and Paul S. Foster
}

We are grateful for an excellent opportunity to expand on our recent Review (Eosinophils: changing perspectives in health and disease. Nature Rev. Immunol. 13, 9-22(2013) $)^{1}$ that has been provided by the correspondence from Carl Persson and Lena Uller (Primary lysis of eosinophils as a major mode of activation of eosinophils in human diseased tissues. Nature Rev. Immunol. http://dx.doi.org/10.1038/ nri3341-c1 (2013))2. To recap: researchers in the eosinophil field have defined three main mechanisms of degranulation. The first, exocytosis, in which cytoplasmic granules fuse with the plasma membrane and release all of their contents into the extracellular space en masse, is not typically observed in vivo. A second type of degranulation, piecemeal degranulation, is a process that was first defined by the electron microscopic observations of Dvorak and colleagues ${ }^{3}$, who noted that, similarly to what had been observed for basophils, eosinophil granules do not empty as completely or as explosively as would be anticipated if exocytosis were taking place ${ }^{4}$. As discussed in detail in the original Review ${ }^{1}$, recent studies by Spencer, Melo, Weller and colleagues ${ }^{5,6}$ have identified tubulovesicular subcellular structures and have elucidated receptorchaperone systems that are the among the important features underlying piecemeal degranulation.

However, a third mechanism, which was covered more briefly, is cytolysis. Cytolylsis is classified as a form of degranulation because, as was recently shown, it is not purely 'death' but a way for eosinophils to live on functionally and to have an important and ongoing immunomodulatory impact on eosinophil-enriched organs and tissues.
The results of eosinophil cytolysis specifically, the release of intact granules are not new findings. There have been many reports of free granules in tissues found in conjunction with eosinophil-associated diseases, including allergic rhinitis, bronchial asthma, atopic dermatitis, urticaria and eosinophilic esophagitis ${ }^{7}$. However, it was not clear what role these granules had, if any, in promoting health or disease. This issue was addressed by the recent work of Neves, Moqbel, Weller and colleagues ${ }^{7}$, who made a substantial step forward by showing us that granules could function in a cell-free state and could secrete immunomodulatory mediators. Specifically, granules isolated by subcellular fractionation express the interferon- $\gamma$ (IFN $\gamma)$ receptor and CC-chemokine receptor 3 (CCR3) on their membranes and release the cationic ribonuclease eosinophil cationic protein (ECP) in a dose-dependent fashion in response to either IFN $\gamma$ - or CC-chemokine ligand 11 (CCL11; also known as eotaxin 1)-mediated stimulation via a p38 mitogen-activated protein kinase (MAPK)dependent signalling pathway ${ }^{7}$. Other receptors that have been identified on cell-free eosinophil granules include cysteinyl leukotriene receptor 1 (CYSLTR1), CYSLTR2 and

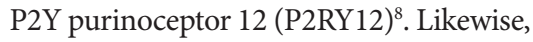
as noted by Uller and Persson, Ueki and colleagues ${ }^{9}$ have reported (since the publication of our Review) that the release of mitochondrial DNA 'nets', which is another form of cytolysis, is also accompanied by the release of intact granules.

Among the important questions that remain to be explored, it is not clear whether the mechanisms of protein trafficking found in intact cells also take place in granules, and/or if individual proteins can be selectively mobilized. Equally interesting, it will be important to determine what conditions in vivo favour piecemeal degranulation and what conditions promote cytolysis (with or without 'net' formation) and release of intact granules. There are several strong recent reviews covering mechanisms of degranulation, including those written by Neves and Weller ${ }^{10}$, and Lacy and Moqbel ${ }^{11}$, for those seeking greater insight into this important and evolving field.

Helene F. Rosenberg is in the Inflammation Immunobiology Section, National Institute of Allergy and Infectious Diseases, National Institutes of Health, Bethesda, Maryland 20892, USA.

Paul S. Foster is at the Priority Research Center for Asthma and Respiratory Diseases,

Hunter Medical Research Institute and School of Biomedical Sciences and Pharmacy, Faculty of Health, University of Newcastle, Newcastle, New South Wales, 2300, Australia.

e-mails: hrosenberg@niaid.nih.gov; paul.foster@newcastle.edu.au

1. Rosenberg, H. F., Dyer, K. D. Foster, P. S. Eosinophils: changing perspectives in health and disease. Nature Rev. Immunol. 13, 9-22 (2013).

2. Persson, C. \& Uller, L. Primary lysis of eosinophils as a major mode of activation of eosinophils in human diseased tissues. Nature Rev. Immunol. http://dx.doi. org/10.1038/nri3341-c1 (2013).

3. Dvorak, A. M. et al. Piecemeal degranulation of specific granules and distribution of Charcot-Leyden crystal protein. Am. J. Pathol. 138, 69-82 (1991).

4. Melo, R. C. $\&$ Weller, P. F. Piecemeal degranulation in human eosinophils: a distinct secretion mechanism underlying inflammatory responses. Histol. Histopathol. 25, 1341-1354 (2010).

5. Melo, R. C., Perez, S. A., Spencer, L. A., Dvorak, A. M. \& Weller, P. F. Intragranular vesiculotubular compartments are involved in piecemeal degranulation by activated human eosinophils. Traffic 6, 866-879 (2005).

6. Spencer, L. A. et al. Cytokine receptor-mkediated trafficking of preformed IL- 4 in eosinophils identifies an innate immune mechanism of cytokine secretion. Proc. Natl Acad. Sci. USA 28, 3333-3338 (2006).

7. Neves, J. S. et al. Eosinophil granules function extracellularly as receptor-mediated secretory organelles. Proc. Natl Acad. Sci. USA 105, 18478-18483 (2008).

8. Neves, J. S., Radke, A. L. \& Weller, P. F. Cysteinyl leukotrienes acting via granule membrane-expressed receptors elicit secretion from within cell-free human eosinophil granules. J. Allergy Clin. Immunol. 125, 477-482 (2010).

9. Ueki, S. et al. Eosinophil extracellular DNA trap cell death mediates lytic release of free secretioncompetent eosinophl granules in humans. Blood 121 2074-2083 (2013).

10. Neves, J. S. \& Weller, P. F. Functional extracellular eosinophil granules: novel implications in eosinophil immunobiology. Curr. Opin. Immunol. 21, 694-699 (2009).

11. Lacy, P. \& Moqbel, R. in Eosinophils in Health and Disease Ch. 7.6 (eds Lee, J. J. \& Rosenberg, H. F.) 206-218 (Elsevier, 2012).

Competing interests statement

The authors declare no competing financial interests. 